\title{
ILCEA
}

Revue de l'Institut des langues et cultures

d'Europe, Amérique, Afrique, Asie et Australie

$20 \mid 2014$

Le conte : d'un art à l'autre

\section{Du récit à la musique : Les Contes de la couleuvre de Pierre Agut}

From Story to Music: The Tales of the Grass-snake by Pierre Agut

\section{Pierre Agut}

\section{OpenEdition}

\section{Journals}

\section{Édition électronique}

URL : http://journals.openedition.org/ilcea/2760

DOI : $10.4000 /$ ilcea.2760

ISSN : 2101-0609

\section{Éditeur}

UGA Éditions/Université Grenoble Alpes

\section{Édition imprimée}

ISBN : 978-2-84310-290-5

ISSN : 1639-6073

\section{Référence électronique}

Pierre Agut, « Du récit à la musique : Les Contes de la couleuvre de Pierre Agut », ILCEA [En ligne], 20 | 2014, mis en ligne le 08 décembre 2014, consulté le 01 mai 2019. URL : http:// journals.openedition.org/ilcea/2760 ; DOI : 10.4000/ilcea.2760

Ce document a été généré automatiquement le 1 mai 2019.

(C) ILCEA 


\title{
Du récit à la musique : Les Contes de la couleuvre de Pierre Agut
}

From Story to Music: The Tales of the Grass-snake by Pierre Agut

\author{
Pierre Agut
}

1 «Il était une fois...» une chef de chœur (Christelle Julien-Issanchou) et un compositeur, moi-même, qui, travaillant au conservatoire municipal du $6^{e}$ arrondissement à Paris, forment le projet de réaliser une œuvre nouvelle pour l'un des chœurs d'enfants de l'établissement.

\section{Contraintes pédagogiques - Sujet - Caractères généraux - Création}

2 Les musiciens (un groupe d'une vingtaine d'enfants âgés de 7 à 9 ans) ont à leur actif une à trois années d'apprentissage musical. Leur pratique vocale se concentre essentiellement sur le chant à l'unisson - plus rarement à deux voix - d'un répertoire appris par cœur, quasiment sans recours à une forme quelconque de partition. Dans la lignée d'une tradition récente mais vivace, nous imaginons une musique à la fois descriptive et narrative dans le sillage de ce qui s'appelle généralement "conte musical», genre répandu dans les répertoires pédagogiques. Le sujet porte sur les trois «Contes de la Couleuvre" des frères Grimm ( $\mathrm{n}^{\circ} 105$ des Contes pour les enfants et la maison) dans la version traduite par Natacha Rimasson-Fertin ${ }^{1}$. Le projet prend ainsi la forme d'une suite de trois pièces narratives n'excédant pas six minutes chacune, dont les parties vocales sont écrites en vue d'être mémorisées par des enfants. L'effectif choral se divise en deux parties chantant tantôt à l'unisson, tantôt en antiphonie ${ }^{2}$-jamais à deux voix superposées. Les pièces demandent la participation d'un(e) pianiste ainsi que d'une voix pour la narration parlée ${ }^{3}$. C'est au cours de l'année 2010 que ces pièces, reprenant le titre "Les Contes de la couleuvre", sont conçues, travaillées et apprises. La création a lieu le 18 mars 2011 à l'Auditorium Saint-Germain à Paris, au cours d'un concert de musique 
vocale donné par les différentes maîtrises et chorales du conservatoire du $6^{\mathrm{e}}$ arrondissement. Parallèlement, les Éditions Delatour publient la partition.

\section{Les textes originaux de Grimm}

3 Dans le texte original, chaque histoire collectée par les Grimm raconte une courte aventure arrivant à un enfant et à une couleuvre, dont voici les résumés.

4 Conte 1 : Tant qu'un petit enfant partage son goûter de pain et de lait avec « la couleuvre de la maison ", l'animal apporte jouets et trésors. Mais lorsqu'à la suite d'une erreur de la mère de l'enfant, l'animal est tué, alors l'enfant dépérit et meurt. Un oiseau de la nuit tresse alors une couronne mortuaire.

5 Conte 2: Une petite fille attire une couleuvre grâce à un morceau de soie. Quand la couleuvre voit ce tissu, elle fait demi-tour, pour revenir avec une couronne, la poser et s'en aller. La fillette s'empare de la couronne. Cela provoque un chagrin mortel à la couleuvre lorsqu'à son retour elle constate que l'objet a disparu. Le conte conclut sur ces mots : « Si la fillette n'avait pas touché à la couronne, la couleuvre lui aurait certainement apporté plus de trésors [...].» (p. 103)

Le conte 3 consiste en un court dialogue farci d'onomatopées entre un enfant qui cherche sa sœur et une couleuvre qui dit n'avoir rien vu.

7 Un saisissant mélange d'étrangeté et de poésie émane de ces formes brèves. Nombreux sont les animaux qui peuplent le monde des contes. Pourtant, les serpents, familiers des contrées mythologiques et bibliques, semblent rares dans les contes occidentaux - aussi bienveillants soient-ils. Dans une note de sa traduction, Natacha Rimasson-Fertin mentionne plusieurs documents témoignant d'une habitude de domestication de couleuvres dans certaines régions d'Europe. La figure du reptile bienveillant fait écho aussi à la couleuvre d'Esculape, animal devin et guérisseur dans l'Antiquité grécoromaine, dont le symbole orne encore aujourd'hui les caducées des professions médicales.

Un réseau de correspondances relie les trois textes. Les deux premiers contes semblent être miroirs l'un de l'autre : la couleuvre apparaît grâce à un objet attirant (« écuelle de lait» ou «foulard de soie bleue»), les pierres brillantes et jouets en or qu'elle apporte dans le premier conte se trouvent rappelés par l'image de la couronne du conte suivant. La dualité enfant/animal paraît structurée par un rapport de confiance qui, s'il est brisé (conte 1) ou non-réciproque (conte 2), entraîne la mort de l'animal, voire celle de l'enfant. $\mathrm{Vu}$ sous cet angle, le dernier conte semble synthétiser les deux précédents:1) le lien entre enfant et couleuvre - qui cette fois dit «non»- est rappelé une dernière fois, 2) l'enfant cherchant sa sœur rappelle l'un ou l'autre des enfants des contes précédents, 3) le détail des «bas rouges » n'est pas sans évoquer le «foulard de soie bleue » du conte précédent.

9 De nombreuses évocations sonores parcourent le texte d'origine. Ainsi, l'appel de l'enfant et le chant de l'oiseau du premier conte, de même que l'appel de la couleuvre et l'aspect « comptine » du dernier conte, agissent comme une «musique en puissance » propre à stimuler l'imagination du musicien. 


\section{L'adaptation des textes en paroles}

$10 \mathrm{Au}$ moment de la composition, les traductions françaises de Natacha Rimasson-Fertin sont adaptées afin d'être chantées - et aisément mémorisées - par des enfants. L'objectif étant de garder les étapes narratives de chaque conte, certains détails sont modifiés. Le deuxième conte retrace le triste sort de la couleuvre, qui « rampa vers la muraille, et, de douleur, [...] se mit à se cogner la tête contre celle-ci, tant que durèrent ses forces, jusqu'à ce qu'elle finisse par mourir». L'adaptation résume cette fin en quelques mots: "De chagrin l'animal se laissa dépérir ». De même, le premier conte inclut l'appel de l'enfant sous une forme proche de la comptine :

Couleuvre, couleuvre, viens vite,

Viens vite, petite bête jolie.

Ton morceau de pain tu auras,

De lait tu te régaleras.

Cette comptine se trouve adaptée ainsi :

Viens couleuvre, viens couleuvre jolie! (bis)

Viens vite! Couleuvre, viens vite!

Viens couleuvre, viens couleuvre jolie! (bis)

Lape, lape, lape, lape le lait ! (bis)

Le lait, le lait ${ }^{4}$ !

11 À travers ces deux exemples, on s'aperçoit que le nombre de mots a tendance à diminuer, tout en gardant les traits principaux du récit. Généralement un ou deux termes importants sont conservés de façon à exprimer l'essentiel de l'idée à travers la mise en musique. De plus, les termes sont choisis et combinés en fonction des possibilités sonores qu'ils offrent. En gommant les détails narrés chez Grimm tout en s'adaptant à la phrase musicale, « de chagrin/l'animal/se laissa/dépérir » épouse un rythme d'anapeste ${ }^{5}$ présent tout au long de la pièce en le reproduisant sur quatre groupes de trois syllabes. L'allitération «lape - le - lait", à la lisière entre signification et sonorité, trouve un prolongement dans l'utilisation du claquement de langue, lui-même à la frontière entre articulation vocale et jeu rythmique pur.

D'expérience, nous savons qu'un texte recevant de la musique diffère d'un texte littéraire se suffisant à lui-même. Nous avons ainsi réalisé des paroles restant intelligibles pour le public lors de l'interprétation. Ce texte reste économe en moyens littéraires : des mots et des phrases courtes, une préoccupation rythmique, l'emploi de syllabes ouvertes, de façon à informer le public des lignes du récit sans gêner le développement musical. Le but de cette œuvre est de raconter trois histoires intégralement en musique et non avec accompagnement/interludes/etc.: le texte ainsi fabriqué reste l'un des moyens de construction et non le but ultime.

Contrairement à la numérotation des Grimm, chaque récit reçoit un titre propre et les trois contes se nomment dans l'ordre: "L'écuelle de lait», «La couleuvre et la couronne », « Dialogue ».

\section{L'élaboration des formes musicales}

Chaque contenu narratif engendre une forme musicale. Pour le premier conte, une forme « couplet-refrain » est introduite par une narration parlée et conclue par un épisode sans 
tempo. Schématiquement voici comment la forme s'organise en regard des différentes péripéties :

Introduction parlée puis parlé-rythmé : « Il était une fois, un petit enfant... »

Refrain : «Viens couleuvre...»

Couplet $1:$ : La couleuvre venait $[. .$.$] et apportait toute sorte de trésors...»$

(Refrain)

Couplet 2 : «Petite bébête, mange aussi le pain. »

(Refrain)

Couplet 3 : « La mère sortit bien vite, avec une bûche tua l'animal. »

Refrain instrumental, avec commentaire du chœur : « L'enfant changea... »

Épilogue sans tempo : "L'oiseau ramassa des feuilles et des branches... »

15 Pour le deuxième conte, une forme strophique avec interludes au piano est interrompue et conclue par le narrateur, ce qui donne en regard des textes:

(Sans tempo)

Strophe $1:$ «Une fille orpheline [...]. »

(Sans tempo)

Strophe $2:$ « La couleuvre apporte une couronne [...]. »

(Sans tempo)

Strophe 3 (tronquée) : « La couronne a disparu!»

Interruption et conclusion : «Qui sait si la couleuvre [...]. »

En raison de son aspect originel proche de la comptine et de son caractère quelque peu absurde, le dernier conte est conçu comme un dialogue entre les deux chœurs, entrecoupé de sections d'improvisation. La forme est en résumée de la manière suivante :

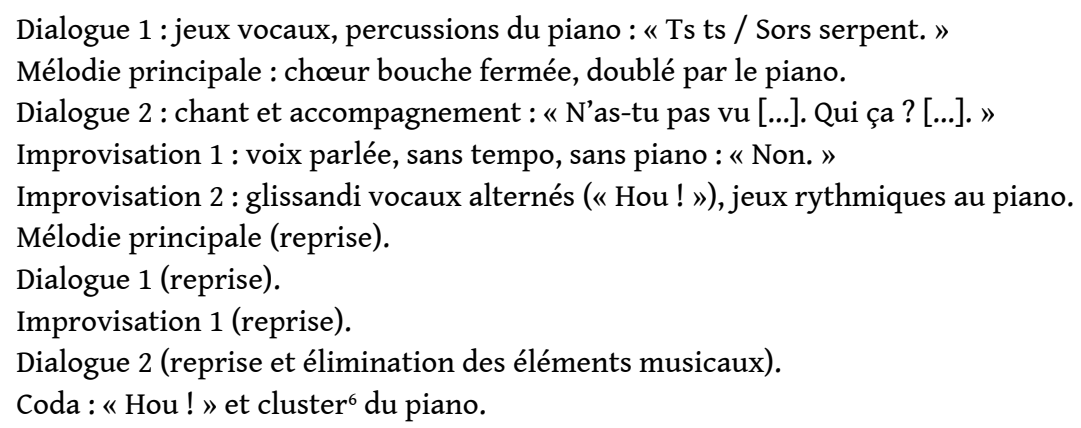

Dans ce dernier conte, l'organisation formelle prend ses distances avec les questions de narration. Le propos littéraire se réduit, la musique suit ses propres chemins. L'architecture musicale s'élabore essentiellement à partir d'éléments sonores. Le développement de l'aspect dialogué et la généralisation des jeux d'onomatopées et de sonorités conduit à l'adoption d'un plan dit "centré » (ou «en arches»). Le centre de gravité, à la fois œil de cyclone et point culminant, réside dans l'improvisation 2. Ce qui précède le centre est récapitulé ensuite dans un ordre différent, en alliant réinterprétation des événements musicaux et goût pour la répétition correspondant aux attentes - supposées ? - du public. Un bref rappel du centre de gravité clôt la pièce.

\section{Élaboration d'une esthétique}

Selon notre projet initial, notre but était non d'habiller de sons des histoires ou bien de les mêler de chansons et intermèdes, mais de créer des récits intégralement mis en musique. 
19 En raison des origines «populaires » des contes et de la destination pédagogique de la musique, les parties vocales adoptent un style populaire réinterprété par l'usage des échelles diatoniques, du faible ambitus vocal, des répétitions mélodiques et des jeux d'antiphonie. Cependant, ces compositions s'ancrent dans un monde contemporain par les nombreux éléments de musiques savantes qui y sont représentés - généralement au piano : conception polyrythmique, effets de timbres, harmonisations dissonantes, passage entre un temps strié (présence d'une pulsation perçue) et un temps lisse (suspension ou absence de pulsation perçue).

La musique tend à s'adapter aux images contenues dans le texte, par un aspect parfois imitatif, parfois évocateur. Dans le registre de l'imitation prennent place les claquements de langue évoqués plus haut, situés à la fin du refrain du premier conte après l'expression «lape le lait», mais dans la même pièce nous mentionnerons la présence d'un lointain sifflement dans l'aigu du piano lorsqu'il est question de «l'oiseau de la nuit ». L'évocation musicale se fait plus subtile : les courbes sinueuses du piano résonnant, qui ponctuent les strophes du deuxième conte, pourraient aussi bien évoquer l'arrivée ondulante de la couleuvre que les reflets de la soie bleue attirant l'animal.

\section{Pour conclure}

Lors de cette expérience pédagogique et artistique, les «Contes de la couleuvre » de Grimm ont agi de trois manières, comme source d'inspiration puisqu'ils ont fourni un sujet et des éléments sonores, comme biais de composition puisqu'il a fallu en adapter les traductions, et comme finalité artistique, c'est-à-dire parvenir à un récit en musique. Les Contes de la couleuvre, par le passage d'une narration à une temporalité musicale, témoignent de la recherche de la meilleure adaptation possible entre un contenu narratif, poétique et musical et un discours formel. De cette adaptation, les « Contes » tentent, audelà des préoccupations pédagogiques qui en sont la source, de faire surgir une émotion chez l'auditeur. Bien sûr, nous restons conscients que d'autres manières de composer de la musique pour les enfants existent.

\section{NOTES}

1. Contes pour les enfants et la maison, tome 2, collectés par les Frères Grimm, édités et traduits par

N. Rimasson-Fertin, Paris : Éditions José Corti, 2009, p. 101-104.

2. On appelle antiphonie le fait que deux chœurs (ou deux voix) chantent en alternance.

3. Assurée au moment de la création par Christelle-Julien Issanchou.

4. Entre ces deux exclamations s'insèrent des claquements de langue.

5. Soit deux brèves puis une longue ( $U$-).

6. Un cluster (groupe) est un agrégat de notes voisines, réalisé au piano à une ou deux mains. 


\section{RÉSUMÉS}

D'abord, une chef de chœur (Christelle Julien-Issanchou) et un compositeur (moi-même) formèrent le projet de réaliser une œuvre nouvelle destinée à l'un des chœurs d'enfants du conservatoire municipal du $6^{\mathrm{e}}$ arrondissement de Paris.

Pour le sujet, ce furent les trois «Contes de la Couleuvre » des Frères Grimm ( ${ }^{\circ} 105$ des Contes pour les enfants et la maison) qui retinrent l'attention en raison de leur brièveté, de leur caractère étrange et de leur poésie.

Les trois pièces furent conçues en 2010 pour double-chœur d'enfants, piano et participation d'une narration parlée, travaillées et apprises au même moment par les jeunes musiciens et créées en mars 2011 à l'Auditorium Saint-Germain à Paris. Parallèlement, les Éditions Delatour publièrent la partition.

Chaque histoire de Grimm raconte une courte aventure arrivant à un enfant et à une couleuvre. Plusieurs « effets sonores » déjà présents dans le texte, tels que l'appel de l'enfant et le chant du rossignol du premier conte, l'appel de la couleuvre et l'aspect «comptine » du dernier conte, stimulèrent la composition.

Au moment de la composition, les traductions de Natacha Rimasson-Fertin furent adaptées afin d'être chantées - et aisément mémorisées - par les enfants. Les quelques détails modifiés ou retirés - comme la mort de la couleuvre à la fin du conte $2-$ ne changent pas les lignes principales des récits. De plus, chaque conte reçoit son titre propre : «L'écuelle de lait », «La couleuvre et la couronne ", " Dialogue ».

Chaque contenu narratif engendre une forme musicale. Pour le premier conte, une forme « couplet-refrain » est introduite par la narration parlée et conclue par un épilogue sans tempo. Pour le second, une forme strophique avec interludes au piano est interrompue et conclue par le narrateur. En raison de son aspect «comptine» et de son caractère quelque peu absurde, le dernier conte est conçu comme un dialogue des deux chœurs entrecoupé de sections d'improvisation.

En raison des origines "populaires " des contes et de la destination pédagogique de la musique, les parties vocales sont écrites dans un style populaire réinterprété (échelles diatoniques, jeux d'antiphonie), habillées d'un riche matériel au piano (accords «non-octaviants", conception polyphonique...). En relation au texte, la musique se fait pour partie imitative et descriptive (effets vocaux des contes 1 et 3), évocatrice d'objets (courbes mélodiques pour l'arrivée de la couleuvre sur la soie bleue au conte 2 ) ou de climats émotionnels (l'attente, la joie, le don, le refus, la mort...).

Par la force de leurs courtes histoires et par la richesse de leurs éléments musicaux, Les Contes de la couleuvre forment une œuvre attirante et émouvante, pour les jeunes musiciens et ceux qui les écoutent.

This project was launched by choir conductor Christelle Julien-Issanchou and composer Pierre Agut. They intended to create an original work for one of the children-choirs of the Conservatoire of $6^{\text {th }}$ arrondissement in Paris. The three Tales of the Grass-Snake by the Grimm brothers were chosen ( $\mathrm{n}^{\circ} 105$ from Children's and Household Tales) for their brevity, strangeness and poetical atmosphere.

The three pieces were written in 2010 for double children choir and piano, with the participation 
of a narrator. They were learned by the young musicians and were premiered in March 2011 at the Auditorium Saint-Germain in Paris. The score was published at the same time by the Delatour Publishing House.

Each story by Grimm tells a little adventure happening to a child and a grass-snake. Many "sonorous effects" are already present in the text, like the child-call and the nightingale's song (tale 1), the grass-snake's call and ditty-mood (tale 3) which the composer found particularly inspiring.

During the composition, Natacha Rimasson-Fertin's French translations were adapted in order to be sung -and easily learned- by children. Some details (like the grass-snake's death at the end of tale 2) were changed or removed, without altering of the main plot of the tales. Furthermore, each tale receives its own title: "The Bowl of Milk", "The Grass-Snake and the Crown", "Dialogue".

Each narrative structure gives rise to its specific musical form. In tale 1, the narrator introduces a verse and chorus form, which ends with a free tempo epilogue. In tale 2, the narrator interrupts the strophic form and its piano interludes, and concludes the piece. Due to its part ditty -and absurd mood, the third tale is conceived as a dialogue between the two choirs, interrupted by improvised sections.

Due to the folk origin of the tales and to the pedagogical use of the music, the vocal parts are written in a partially reinvented popular style, through use of diatonic scales and of antiphonic games, supported by rich polyphonic piano part, deprived of octaving chords. Part of the music is at times imitative or descriptive, in relation to the text (see the vocal effects in tales 1 and 3). Part of it is also evocative of specific objects, like the shape of the melody for the snake's arrival on a blue silk in tale 2. Part of it at last, evokes different moods, such as expectation, joy, giving, denial, loss or death.

The power of these short tales and the rich and original elements of the music make the Tales of the Grass-Snake an attractive and stirring work for young musicians and their audiences.

INDEX

Mots-clés : conte, couleuvre, musique, conte musical, chœur d'enfants, piano, paroles, voix

Keywords : tale, grass-snake, music, musical tale, children's choir, piano, lyrics, voices

\section{AUTEUR}

\section{PIERRE AGUT}

Compositeur et professeur des conservatoires de la ville de Paris 